\title{
ARTÍ́CULOS
}

\section{La nanotecnología en el CSIC: transferencia y comercialización de patentes}

\author{
Javier Etxabe, ${ }^{1}$ Javier Maira ${ }^{1}$ y Pedro A. Serena ${ }^{2}$
}

\begin{abstract}
RESUMEN: La nanociencia y la nanotecnología hicieron su aparición en el escenario científico en un momento en el que la economía de España y el sistema español de investigación, desarrollo e innovación experimentaban un fuerte crecimiento. Esta circunstancia propició un notable desarrollo de la nanociencia y la nanotecnología especialmente en el entorno académico universitario y en organismos públicos de investigación como el Consejo Superior de Investigaciones Científicas (CSIC). Sin embargo, este desarrollo no ha tenido reflejo en un aumento igual de importante en la transferencia de conocimiento desde los laboratorios al sector productivo. Es en este contexto en el que el CSIC ha puesto en marcha una serie de acciones, cuyo objetivo ha sido sacar provecho del conocimiento generado en las actividades de $I+D$, mediante su transferencia al sector industrial para llevar al mercado productos y servicios de alto valor añadido. En este artículo se describen estas acciones y se presentan los resultados alcanzados en el sector de las nanotecnologías, en el que se ha hecho un especial esfuerzo en los últimos cinco años.
\end{abstract}

ABSTRACT: Nanoscience and nanotechnology arose in the scientific scene at a time when the economy of Spain and the spanish system of research, development and innovation were experiencing strong growth. This circumstance led to a remarkable development of nanoscience and nanotechnology especially in the academic field including universities and public research institutions as the Spanish National Research Council (CSIC). However, this development has not been reflected in an equally remarkable increase in the transfer of knowledge from laboratory to industry. This is the context in which CSIC has launched a series of actions whose objective was to take advantage of the knowledge generated in R\&D projects, through its transfer to industry to bring out high value products and services to the market. This article describes these actions as well as the results achieved in the field of nanotechnology, in which CSIC has made a special effort in the last five years.

\section{LA LLEGADA DE LA NANOTECNOLOGÍA}

A lo largo de las tres últimas décadas hemos asistido a una paulatina acumulación de conocimientos en el ámbito de la nanociencia, la disciplina que estudia el comportamiento de materiales y dispositivos en la denominada "nanoescala", un territorio que corresponde a las dimensiones comprendidas entre unas décimas y varios centenares de nanómetros (Lindsay, 2009). La nanociencia se fue desarrollando paulatinamente en los laboratorios de los países más avanzados hasta que hace poco más de diez años una serie de circunstancias provocaron una inmensa inyección de recursos económicos con el fin de aprovechar todos los conocimientos acumulados y convertirlos en un instrumento con la capacidad de cambiar nuestro futuro (Pagliaro, 2010).

\footnotetext{
1 Vicepresidencia Adjunta de Transferencia del Conocimiento (VATC). Consejo Superior de Investigaciones Científicas (CSIC). c/ Serrano 142, E-28006-Madrid, España.

2 Instituto de Ciencia de Materiales de Madrid (ICMM). Consejo Superior de Investigaciones Científicas (CSIC). c/ Sor Juana Inés de la Cruz 3, Campus de Cantoblanco, E-28049-Madrid, España. *Correo-e: pedro. serena@icmm.csic.es; tel.: +34913348999.
} 
Se podría afirmar que el año 2000 es la fecha en la que la nanociencia dejó paso a la nanotecnología, pues ese momento corresponde a la puesta en marcha del programa Nanotechnology National Initiative (conocido por su acrónimo NNI) por parte del presidente de los EEUU W. Clinton (NNI, 2012; Kleike, 2009). Este programa ha propiciado, mediante inversiones acumuladas de más de 11,000 millones de dólares, la construcción de nuevos centros de investigación y la financiación de proyectos multidisciplinares y programas formativos. Además, este programa permitió a EEUU alcanzar el liderazgo de este paradigma científicotécnico emergente, planteando desde un principio un modelo en el que los sectores productivos serían los grandes receptores y transformadores del conocimiento generado en universidades y centros de investigación, sentando las bases para una revolución de enormes repercusiones socioeconómicas (Roco, 2001). Desde el lanzamiento del programa NNI, todos los países desarrollados siguieron la senda marcada por los EEUU, planteando iniciativas similares con el fin de no quedar descolgados en la carrera de la nanotecnología (Delgado, 2008, 2009). La Unión Europea incorporó un área temática "Nanotecnología, Materiales y Procesos de Producción" (conocida como NMP) entre las líneas prioritarias de los Programas Marco VI y VII (Comisión Europea, 2004, 2005). La primera consecuencia de todos estos impulsos fue el considerable aumento del número de publicaciones científicas relacionadas con la nanociencia y la nanotecnología (Palmberg, 2009). El porcentaje de las publicaciones relacionadas con estos temas con respecto del total de publicaciones referenciadas por el Science Citation Index (SCI) (Thomson Reuters, 2012) pasó del 2.5\% en el año 2000 al 4.1\% en el año 2007 (OEI, 2009).

En la actualidad la inversión pública conjunta por parte de los gobiernos de todo el mundo se sitúa en los 10,000 millones de dólares anuales y se prevé un crecimiento de un $20 \%$ durante los próximos tres años (Cientifica, 2011). Nos encontramos ante una de las líneas científicas que más financiación está recibiendo a nivel global, con cantidades similares a las dedicadas en el pasado a otros programas como la decodificación del genoma humano o la puesta en marcha de grandes aceleradores de partículas. Esta inversión ha dado lugar a un gran número de patentes en el ámbito de la nanotecnología (Palmberg, 2009, Dang, 2010) y se han llegado a definir descriptores específicos relacionados con la clasificación de patentes en este campo (EPO, 2011). En estos momentos EEUU, Alemania, Francia, China, Japón, Corea del Sur y Taiwán están liderando la actividad en nanotecnología, aunque son los tres últimos países mencionados los que poseen una mayor capacidad de comercialización de productos basados en nanotecnología o "nanoproductos" (Hwang, 2010). Esta actividad va a propiciar un gigantesco mercado (Palmberg, 2009) y este potencial económico ha dado lugar a varios índices bursátiles que agrupan empresas emergentes que cotizan en mercados de valores internacionales (Merrill Lynch, 2004; ISE, 2009).

\section{LA SITUACIÓN DE LA NANOTECNOLOGÍA EN ESPAÑA}

A la vez que la nanociencia se consolidaba a nivel mundial, España comenzó una lenta convergencia con los países más avanzados de su entorno desarrollando una economía basada en industrias como la agroalimentaria, la automoción, la fabricación de máquina-herramienta y la petroquímica, y en el sector terciario, donde destacaban el turismo y el sector financiero. Este proceso convergente también tuvo lugar en el ám- 
bito de la ciencia por lo que el número de recursos, investigadores, institutos, proyectos y publicaciones comenzaron a crecer con un buen ritmo. Todo este crecimiento tuvo lugar en el marco legal proprocionado por la Ley de Fomento y Coordinación General de la Investigación Científica y Técnica, comúnmente denominada como "Ley de la Ciencia”, promulgada en el año 1986 (Ley de la Ciencia, 1986), que ha permitido el mantenimiento de convocatorias estables insertadas en un sistema plurianual de planificación mediante los Planes Nacionales de Investigación, Desarrollo e Innovación $(\mathrm{I}+\mathrm{D}+\mathrm{I})$. Otro aspecto que no debe olvidarse es la entrada de España en la Comunidad Económica Europea (ahora Unión Europea) en el año 1986. Dicha incorporación ha permitido el acceso a recursos con los que se han mejorado, entre otras cosas, infraestructuras científicas y programas de formación. Este contexto permitió a la ciencia española dar un salto cualitativo en la generación de conocimiento de calidad, logrando que España ocupe actualmente un puesto de privilegio entre los países que más publicaciones científicas producen. En el año 2010, el gasto en actividades de investigación y desarrollo (I+D) representaba el 1.39\% del producto interior bruto (PIB) y las publicaciones en las que participaban instituciones españolas constituían el 3.55\% de la producción científica mundial (ICONO, 2012).

Sin embargo, como se comentará más adelante, este crecimiento en número de publicaciones no se ha visto reflejado en un incremento similar en cuanto a los conocimientos protegidos y transferidos al sector industrial. Como ejemplo, podemos mencionar que el número de patentes españolas registradas en 2010 bajo el Tratado de Cooperación en Patentes (PCT, Patent Cooperation Treaty) representaban el 1.1\% del total mundial (WIPO, 2011A). Del mismo modo, en el año 2006 los productos españoles sólo representaban el $0.52 \%$ de la cuota total mundial de exportaciones de bienes de alta tecnología (Meri, 2009). Este fracaso relativo de nuestro sistema de I+D+I para transferir el conocimiento desde los laboratorios hasta los mercados ha sido una de las razones para promulgar una nueva Ley de la Ciencia en el año 2011 (Ley de la Ciencia, 2011) y para poner en marcha por primera vez una completa Estrategia Estatal de Innovación (E2I, 2011).

Por lo tanto, a la vez que la nanociencia y la nanotecnología hacían su aparición en el espacio científicotecnológico, España emergía con cierto empuje en la escena científica internacional. De esta forma, la nanociencia y la nanotecnología desarrolladas en centros de investigación españoles han podido seguir un camino bastante similar al trazado por los países de su entorno (Correia, 2006). En esta coyuntura de consolidación del sistema científico español tuvo lugar la aparición de la iniciativa NanoSpain, la Red Española de Nanotecnología (NanoSpain, 2012), que desde el año 2000 aglutina una gran cantidad de grupos de investigación, tanto del sector público como del privado. En la actualidad, la Fundación Phantoms coordina la red NanoSpain (Phantoms, 2012). Gracias a la actividad desarrollada por NanoSpain, junto con otras iniciativas lanzadas por diferentes grupos e instituciones, la nanotecnología tuvo la consideración de Acción Estratégica en los Planes Nacionales de I+D+I que se desarrollaron durante los periodos 2004-2007 (PNIDI, 2004; Serena, 2009A) y 2008-2011 (PNIDI, 2008). Además, el Programa Ingenio 2010 (Ingenio, 2010) proporcionó recursos adicionales que favorecieron el desarrollo de ambiciosos proyectos de investigación más cercanos a la industria, a través de varios Consorcios Estratégicos Nacionales de Investigación Tecnológica (CENIT), en los que la nanotecnología desempeña un papel clave para reactivar o mantener la capacidad de diferentes sectores industriales a medio y largo plazo (OPTI, 2008). Todos estos 
impulsos dieron lugar a la puesta en marcha de numerosos proyectos colaborativos, centros de investigación y grandes infraestructuras singulares, como el sincrotrón ALBA (ALBA, 2012) o el Laboratorio Ibérico Internacional de Nanotecnología (INL) (INL, 2012), consiguiendo una notable consolidación de la nanotecnología española (Phantoms, 2011A).

Este destacable crecimiento del sector "nano", a pesar de tener algunos problemas de coordinación pendientes de su resolución (Serena, 2009B; Chacón, 2011), permitió que en el periodo 1999-2010 España ocupase la décima posición mundial en cuanto a producción científica en este ámbito (Hullmann, 2006; López, 2011; INIC, 2012). Otros estudios (Palmberg, 2009) sitúan a España en la posición undécima en el periodo 1991-2007, indicando que en el periodo 1996-2006 España ocupó la séptima posición mundial en cuanto a la tasa anual de crecimiento de las publicaciones en nanotecnología. En cualquier caso se puede afirmar que las iniciativas y programas que se pusieron en marcha sirvieron para mantener la estela de los países más avanzados. Esta posición de privilegio en cuanto a la producción científica no tiene, sin embargo, una contrapartida adecuada en cuanto a la solicitud, registro y licencia de patentes ni en la generación de nuevas empresas de base tecnológica (NEBTs). En un completo análisis del periodo 1991-2008, España no figura entre las quince naciones que lideran la clasificación de solicitudes acumuladas de patentes relacionadas con la nanotecnología (Dang, 2010). En un estudio patrocinado por la Comisión Europea (Palmberg, 2009), España ocupaba la posición 19 en cuanto al número de patentes acumuladas hasta el año 2005. Cuando se representa el número de patentes frente al número de publicaciones normalizado según el PIB de cada país, España se sitúa en las últimas posiciones a nivel mundial, con una patente por cada 300 artículos, mientras que Irlanda, que gasta 5.6 euros por persona y año en nanotecnología, produce una patente por cada 18 artículos (Noyons, 2003). En cuanto a la creación de NEBTs, recientes informes (Chacón, 2011; Phantoms, 2011B; Correia, 2012) revelan que el número de empresas españolas que están implicadas en el desarrollo y el uso de la nanotecnología es muy escaso en comparación con lo que ocurre en los países que mantienen niveles similares de producción científica.

En resumen, frente a una investigación científica en nanotecnología muy productiva en España, su vertiente aplicada es muy escasa y las relaciones con las empresas del entorno son poco significativas. Este hecho es similar a la situación planteada en muchos países de Iberoamérica, donde la producción industrial se basa en tecnologías de un valor añadido medio o bajo, generalmente extranjeras, y el uso de procesos, materiales o dispositivos basados en tecnología puntera protegida mediante patentes es muy escaso. Estos síntomas no sólo se perciben en el ámbito de la nanotecnología sino que, como ya se ha mencionado anteriormente, corresponden a una situación endémica del sistema ciencia-empresa-sociedad español que ha demostrado una preocupante falta de canales efectivos para convertir las grandes cantidades de conocimientos generados en universidades, organismos públicos de investigación y centros tecnológicos en bienes y servicios (Rodríguez, 2007). Precisamente, los nuevos instrumentos antes mencionados, la Ley de la Ciencia (Ley de la Ciencia, 2011) y la Estrategia Estatal de Innovación (E2I, 2011) se han diseñado para corregir esta situación, aunque en el corto y medio plazo las condiciones económicas serán el factor relevante que condicione el desarrollo del sistema de I+D+I (Correia, 2009). 


\section{El CSIC, REFERENTE ESPAÑol E IBEROMERICANo de LA NANOTECNOLOGía}

Esta sección analiza el papel que ha desempeñado la Agencia Estatal Consejo Superior de Investigaciones Científicas (CSIC) en el desarrollo de la nanotecnología en España. El CSIC (CSIC, 2012) es la mayor institución pública española dedicada a la investigación y la tercera de Europa tras el Max Planck de Alemania y el CNRS francés. En la actualidad, la Agencia Estatal CSIC está adscrita al Ministerio de Economía y Competitividad (MINECO, 2012), a través de la Secretaría de Estado de Investigación, Desarrollo e Innovación. Su misión fundamental es el fomento, la coordinación, el desarrollo y la difusión de la investigación científica y tecnológica de carácter pluridisciplinar, con el fin de contribuir al avance del conocimiento y al desarrollo económico, social y cultural, así como a la formación de personal especializado. Sobre el CSIC también recae la responsabilidad de gestionar un conjunto de importantes infraestructuras: buques oceanográficos, grandes telescopios, reservas biológicas, estaciones polares, centros de nanofabricación, museos, la mayor red de bibliotecas especializadas de España, etc. Merece la pena destacar que entre las funciones del CSIC se encuentran el asesoramiento de entidades públicas y privadas en cuestiones científico-técnicas, la transferencia de resultados al sector empresarial, la contribución a la creación de NEBTs, el fomento de la cultura científica de los ciudadanos y la representación científica de España en el ámbito internacional. Todos estos datos están disponibles en la página web del CSIC (CSIC, 2010).

El CSIC desempeña un papel central en la política científica y tecnológica, ya que abarca desde la investigación básica a la transferencia del conocimiento al sector productivo. Para alcanzar éxito en esta misión, el CSIC cuenta con más de 13,500 trabajadores (cifras de 2010), de los cuales un poco más de 4,400 son investigadores doctores (tanto permanentes como contratados) y otros 3,100 son investigadores en formación. Las actividades de investigación se desarrollan en 75 centros de investigación propios, 53 centros mixtos (creados mediante convenios con universidades y otras instituciones) y 5 grandes centros de servicios. Aunque los investigadores del CSIC sólo representan el $6 \%$ del personal dedicado a actividades de investigación y desarrollo en toda España, producen aproximadamente el 20\% de la producción científica nacional, es decir casi el $0.7 \%$ de la producción científica mundial.

Una de las grandes ventajas competitivas del CSIC es su carácter multidisciplinar y multisectorial ya que cubre todos los campos del conocimiento. Los diferentes institutos y sus líneas temáticas se organizan en torno a ocho áreas científico-técnicas: (i) Humanidades y Ciencias Sociales, (ii) Biología y Biomedicina, (iii) Recursos Naturales, (iv) Ciencias Agrarias, (v) Ciencia y Tecnologías Físicas, (vi) Ciencia y Tecnología de Materiales, (vii) Ciencia y Tecnología de Alimentos, y (viii) Ciencia y Tecnologías Químicas. Precisamente este carácter multidisciplinar es clave para entender el gran desarrollo que la nanociencia y la nanotecnología han tenido en esta institución.

Se puede considerar que la historia de la nanotecnología en el CSIC arranca a principios de la década de los años 90 con el desarrollo de diversas iniciativas entre las que podemos destacar la puesta en marcha del Laboratorio de Física de Sistemas Pequeños y Nanotecnología (LFSPN, 2012). Posteriormente, siguiendo las directrices del Plan Nacional de I+D+I 2004-2007 (PNIDI, 2004), el CSIC implementó el Plan de Actuación Institucional del CSIC para el periodo 2006-2009 (CSIC, 2006A) que contenía un programa estratégico denominado Eje de Nanociencia y Nanotecnología (CSIC, 2006B), también denominado "Eje Nano". Este programa contemplaba una serie de 
actuaciones que han permitido poner en marcha nuevos institutos, con las consiguientes dotaciones de infraestructuras, plazas, y proyectos internos de investigación. El Eje Nano contaba con la participación de investigadores pertenecientes a casi una treintena de institutos del CSIC, en los que se desarrollaban actividades de investigación que comprendían desde la síntesis y caracterización de nanomateriales y nanopartículas hasta su aplicación en estructuras o en el diseño de dispositivos electrónicos y sensores, pasando por las aplicaciones en nanomedicina. Hay que mencionar que el CSIC fue pionero en España en la incorporación de grupos de investigación interesados en el impacto social y la gobernanza de las nanotecnologías (Echeverría, 2005).

El potencial actual del CSIC en nanociencia y nanotecnología se pone de manifiesto en la participación de los grupos de esta institución en la Red NanoSpain. En la actualidad 67 de los 332 grupos que forman la red NanoSpain, es decir un poco más del 20\%, pertenecen a algún instituto o centro del CSIC (NanoSpain, 2012; Serena, 2009B). Estos grupos se ubican en 24 institutos de investigación entre los que podemos destacar, por número de grupos e investigadores, los siguientes: el Instituto de Ciencia de Materiales de Madrid (ICMM), el Instituto de Catálisis y Petroleoquímica (ICP), el Instituto de Microelectrónica de Madrid (IMM), el Centro de Investigaciones en Nanociencia y Nanotecnología (CIN2), el Instituto de Ciencia de Materiales de Barcelona (ICMAB), el Instituto de Ciencia de Materiales de Aragón (ICMA) y el Instituto de Microelectrónica de Barcelona (IMB). Según los datos proporcionados por NanoSpain, los grupos "nano" del CSIC están ubicados mayoritariamente en Madrid y Barcelona, tal y como se muestra en la figura 1a. Esta concentración tiene su origen en dos factores: por un lado la herencia de una estructura bastante centralizada en la que el peso de los centros ubicados en Madrid era considerable y, por otro, la existencia de una aglomeración de centros dedicados a nanotecnología en el entorno del Campus de Excelencia Internacional CEI UAM+CSIC, formado mediante una alianza entre la Universidad Autónoma de Madrid, el CSIC, el Parque Científico de Madrid, el CIEMAT, el Centro IMDEA-Nanociencia de la Comunidad Autónoma de Madrid y varios ayuntamientos de la zona norte de Madrid (CEI, 2012). Esta concentración de institutos en Madrid, junto con otra formada en el entorno del Campus de la Universidad Autónoma de Barcelona y del Sincrotón ALBA (ALBA, 2012) son, sin duda alguna, los dos grandes "polos nanotecnológicos" que el CSIC ha configurado a lo largo de la última década. Sin duda alguna, estos clusters científico-tecnológicos, ubicados en regiones con industrias de alta intensidad tecnológica, constituyen una gran apuesta del CSIC para competir con otras iniciativas similares que se han impulsado en otros países de Europa, EEUU, China o Japón.

En cuanto a las líneas de investigación que se desarrollan en el CSIC, se puede decir que son bastante similares a las que desarrollan, en su conjunto, el resto de grupos de investigación del resto de las universidades y centros tecnológicos de España. En la figura 1b se muestran las actividades temáticas en las están involucrados los grupos del CSIC que son miembros de la red NanoSpain. Se constata que las áreas temáticas con más peso están relacionadas con la investigación en materiales nanoestructurados, nanopartículas, catalizadores, y materiales y dispositivos de utilidad en biotecnología y medicina. Por otra parte las investigaciones en nanomagnetismo, espintrónica, nanotubos de carbono, grafeno, y materiales y dispositivos de interés en fotónica y electrónica tienen un peso medio. Las actividades relacionadas con nanofabricación, instrumentación o metrología son las que poseen una menor representación. 
FIGURA 1. (a) Distribución geográfica de los grupos del CSIC que actualmente son miembros de la red NanoSpain. (b) Distribución temática de las actividades de investigación en nanociencia y nanotecnología de dichos grupos, considerando que cada grupo de investigación puede definir al menos tres áreas de actividad. Fuente: elaboración propia a partir de los datos de la Red NanoSpain (NanoSpain, 2012).
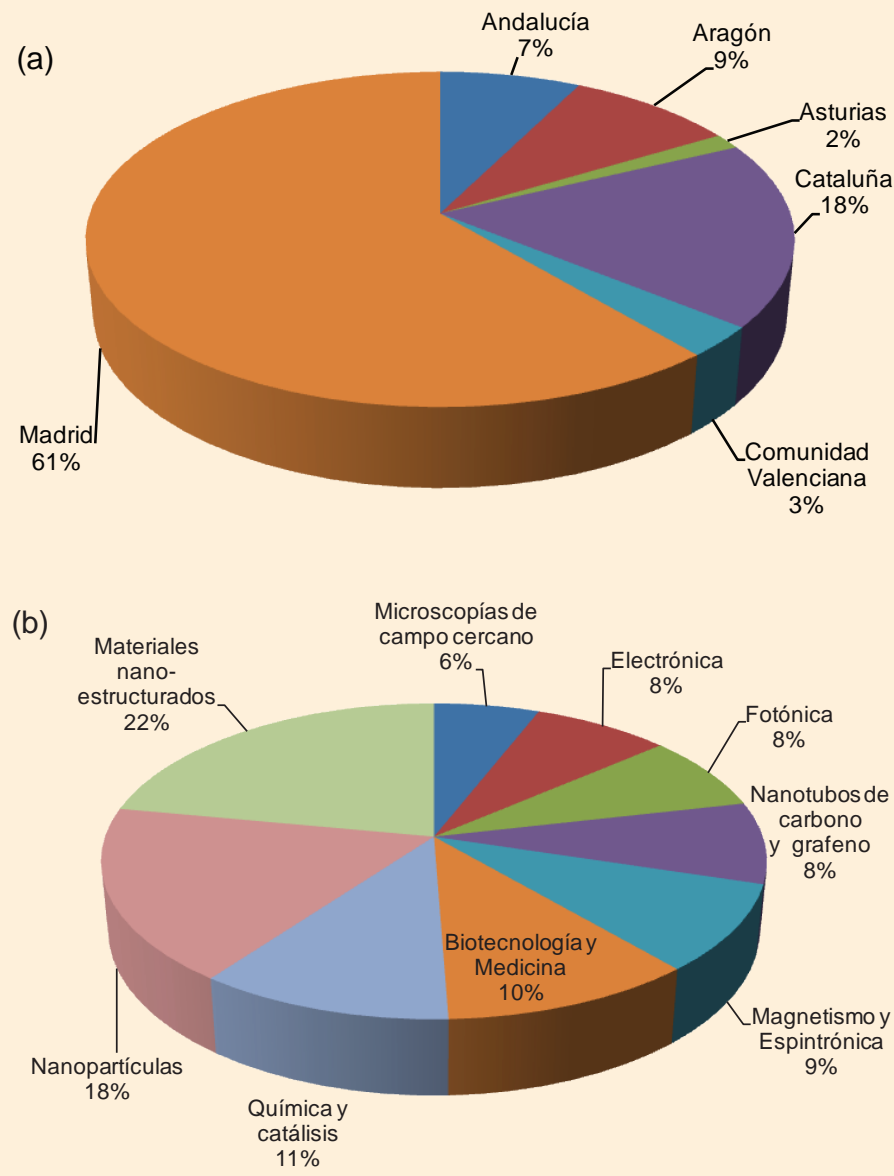

Si se comparan los datos de la figura $1 \mathrm{~b}$ con los indicadores de la estructura de la industria española por ramas de actividad (MINETUR,2010) se observan ciertas analogías. Por ejemplo, el sector industrial de la fabricación de productos basados en materiales metálicos y no metálicos tiene un peso conjunto del $10.1 \%$, el sector de la automoción, que también es un gran consumidor de materiales avanzados, tiene un peso del 9.81\%, y la industria química (que incluye la actividad farmacéutica) tiene un peso del $9.63 \%$. Estos sectores industriales coinciden razonablemente con las temáticas en las que los grupos del CSIC están desarrollando su actividad en el ámbito de la nanotecnología. Por otro lado, las industrias vinculadas con la electrónica y las tecnologías de las telecomunicaciones apenas tienen una contribución del 1.3\% en el conjunto de la producción industrial española. Este menor peso también tiene re- 
flejo en la menor proporción de los grupos del CSIC que mantienen actividad en estas temáticas. Por otro lado, estos indicadores de actividad industrial muestran que la industria de la alimentación y bebidas tiene un peso del $18.2 \%$ sobre el total de la producción industrial española. Este peso no se constata en la figura 1b, pero se debe tener en cuenta que el CSIC cuenta con un área de investigación relacionada con estos temas y que el impacto de la nanotecnología en estos sectores es aún incipiente. Con estos datos se constata que la actividad investigadora en el ámbito de la nanotecnología por parte de los grupos del CSIC debería tener cierto encaje temático en el tejido industrial español. Sin embargo, como se verá en las secciones siguientes este encaje no es lo suficientemente efectivo como para lograr una transferencia eficaz de la nanotecnología al sector productivo.

Ha quedado claro el papel del CSIC como elemento clave de la articulación del sistema de I+D+I español, pero no debemos olvidar que el CSIC, abierto a la colaboración con entidades extranjeras, también es una referencia en el contexto iberoamericano. En la actualidad el CSIC es la institución iberoamericana con mayor presencia en el SCI participando en $11.8 \%$ de la producción iberoamericana en nanotecnología (OEI, 2009). A su vez es la entidad que mantiene más colaboraciones con universidades y organismos iberoamericanos en el campo de la nanociencia y la nanotecnología (OEI, 2009). Como se analiza en las siguientes secciones, esta posición privilegiada también se pone de manifiesto en el ámbito de las patentes en nanotecnología, ya que en Iberoamérica el principal titular es el CSIC, superando en más de tres veces a la siguiente entidad (OEI, 2009).

\section{LA TRANSFERENCIA DE TECNOLOGía EN EsPAÑA}

En el año 1980, la Ley de Patentes y Marcas de los EEUU sufrió profundas modificaciones, entre las que cabe destacar la enmienda promovida por los senadores B. Bayh y R. Dole (Mowerya, 2002). Siguiendo la filosofía de estas modificaciones, tiene lugar en España la promulgación de la Ley de Patentes 11/1986, de 20 de marzo, que reconoce como invenciones laborales aquellas que surjan de los resultados de investigación de las universidades o centros públicos de investigación y, por tanto, reconoce a estas entidades como titulares de los derechos de propiedad industrial relacionados. Al mismo tiempo, la anteriormente mencionada "Ley de la Ciencia" de 1986 (Ley de la Ciencia, 1986) establece las colaboraciones de los centros públicos de investigación con el sector empresarial en el marco de las relaciones privadas y a precio de mercado.

Aprovechando el nuevo marco jurídico y la legislación que confería autonomía administrativa a las universidades, a finales de los ochenta se crean en España las primeras Oficinas de Transferencia de Resultados de Investigación (OTRIs) tanto en las universidades como en los Organismos Públicos de Investigación (OPIs), catalizadas por la Oficina de Transferencia de Tecnología de la Secretaría General del Plan Nacional de Investigación, Desarrollo e Innovación, que se creó en 1989 (Represa-Sánchez, 2005). Aunque el marco de responsabilidades y actividades de las OTRIs ha sido amplio y ambicioso desde sus inicios, la mayor parte de las mismas concentraron, en una primera etapa, sus recursos en la gestión de proyectos de investigación -nacionales y europeos- mientras que la gestión de la protección de resultados de investigación y el asesoramiento y negociación de contratos de I+D+I se empieza a consolidar a fi- 
nales de la década de 1990. Sin embargo, las acciones proactivas de promoción tecnológica de los resultados de investigación (patentes) y la atención directa de demanda tecnológica de empresas y las consiguientes negociaciones y firmas de contratos de licencia y de contratos de investigación de valorización asociados, como estrategia consolidada en una OTRI, son mucho más recientes.

En su conjunto, las universidades españolas y el CSIC, el OPI que más conocimiento genera, presentan una evolución positiva en los indicadores de transferencia de conocimiento a lo largo de las dos últimas décadas: número de solicitudes de patentes de prioridad y solicitudes internacionales PCT (Patent Cooperation Treaty), número e ingresos por contratos de investigación (el 11\% de la investigación financiada en las universidades procede de la contratación con empresas e instituciones), contratos de licencia y creación de nuevas empresas de base tecnológica. Según datos de la Organización Mundial de la Propiedad Intelectual (OMPI), España ocupa la posición número 15 en cuanto a solicitudes de patentes PCT, con un total de 1725 patentes PCT en el año 2010, lo que representa un aumento del $12 \%$ con respecto del año 2009 , a pesar de la actual crisis económica (WIPO, 2011A; WIPO, 2011B). Este número de patentes representó una cuota del 1.1\% del total de patentes PCT. Sin embargo, la posición de España en el ranking internacional empeora considerablemente cuando se utilizan como indicadores el número de patentes normalizado por el número de habitantes o por producto interior bruto (PIB) (Altran, 2012).

Por otro lado, la transferencia de conocimiento mediante la licencia de patentes se ha incrementado en España en los últimos 5 años. Sin embargo, los indicadores españoles asociados a promoción tecnológica y licencias no están equilibrados con respecto a los asociados a la contratación de proyectos con empresas, por lo que van a precisar que las OTRIs españolas adquieran un tamaño medio mayor (en la actualidad muchas de ellas son de tamaño muy pequeño y realizan pocas actividades en este campo), una mayor profesionalización y una menor temporalidad de los recursos humanos, y una menor dependencia de recursos económicos externos. Esta actividad se deberá desarrollar en un contexto de reducción de recursos económicos, ya que el gasto en investigación, desarrollo e innovación tecnológica por parte de las empresas españolas ha caído en los últimos años tras una fase continuada de crecimiento durante el período 2000-2008. Los efectos negativos de la crisis también se han notado en la contratación de servicios o proyectos de $\mathrm{I}+\mathrm{D}+\mathrm{I}$ por parte de las empresas a universidades y OPIs (Red OTRI, 2010). Apenas un 2\% de las empresas colaboran con universidades u organismos públicos de investigación y sólo un 10\%, en el mejor de los casos, de las consideradas empresas innovadoras (INE, 2010). Una prueba de la falta de empresas interesadas en el desarrollo de actividades de I+D+I es la elevada proporción de patentes que en España proceden de entidades académicas públicas, el $10-15 \%$ del total de patentes, en comparación con otros países como, por ejemplo, EEUU donde éstas no representan más del 1-2\%.

Toda esta situación se acentúa en el sector de la nanotecnología en España, donde operan muy pocas empresas, de pequeño tamaño y de muy reciente creación (Chacón, 2011; Phantoms, 2011B; Correia, 2012). Hasta el año 2005 las patentes españolas nanotecnológicas con solicitud en la Oficina Europea de Patentes (EPO; European Patent Office) (EPO, 2012) por parte de empresas representaban menos del 15\% del total mientras que en Europa la media es el $66 \%$ y hay países como Holanda, Suecia, Austria, Alemania y Japón en los que las empresas poseen más del 75\% de las patentes en nanotecnología (Palmberg, 2009). 


\section{Procedimientos de transferencia de tecnología en el CSIC}

Para lograr parte de los objetivos encomendados al CSIC, las actividades tendentes a incrementar la transferencia de tecnología al sector productivo han ido adquiriendo un mayor protagonismo. En el año 1985, se puso en marcha la Oficina de Transferencia de Tecnología (OTT) del CSIC, que en el año 2008 adquirió un mayor rango administrativo, pasándose a denominar Vicepresidencia Adjunta de Transferencia de Conocimiento (VATC). Éste es un claro indicador de la relevancia que esta actividad ha ido adquiriendo en el CSIC. La función principal de la VATC del CSIC es acercar las capacidades científicas y los logros tecnológicos del CSIC a todos los sectores socioeconómicos a escala nacional e internacional (empresas privadas o públicas, otros organismos públicos, fundaciones privadas y públicas, centros tecnológicos, etc.), con la finalidad de lograr que la investigación que realiza el CSIC se transforme en bienestar social, económico y cultural. Para ello pretende:

- Transferir los resultados de investigación y la tecnología desarrollada en el CSIC a la empresa privada para su explotación en el mercado.

- Transferir el conocimiento generado en el CSIC a la sociedad.

- Dar a conocer a las empresas e instituciones públicas los beneficios económicos que pueden derivarse de sus relaciones con los diversos centros e institutos del CSIC.

- Informar a los investigadores del CSIC de las necesidades que se detectan en las empresas y en las instituciones públicas.

- Impulsar la creación de Nuevas Empresas de Base Tecnológica (NEBTs) surgidas a partir del conocimiento generado en el CSIC.

Para poder afrontar estos retos con eficacia y eficiencia, la VATC, dispone actualmente de tres departamentos, bien sincronizados entre sí, con funciones bien diferenciadas:

- Departamento de Contratos y Calidad, encargado de la preparación y gestión de determinadas convocatorias públicas relacionadas con la transferencia de conocimiento, y la preparación de contratos de I+D y apoyo tecnológico entre grupos del CSIC y otras entidades. Este departamento también se ocupa de asegurar el cumplimiento de los compromisos de calidad en la VATC.

- Departamento de Protección de los Resultados cuyo objetivo fundamental es la protección, habitualmente mediante patente, de los resultados que tengan aplicación en el mercado obtenidos a partir de la actividad en I+D en los diferentes centros del CSIC.

- Departamento de Comercialización, donde se lleva a cabo la evaluación y promoción de dichos resultados protegidos, y la negociación con las empresas interesadas en los mismos para una correcta licencia de los derechos de explotación de la patente a la empresa asegurando así su posterior desarrollo y puesta en el mercado.

Hasta principios de 2012 la VATC disponía de un cuarto departamento, la Unidad de Apoyo a la Creación de Empresas (UACE), encargado de potenciar la creación de 
nuevas empresas de base tecnológica (NEBTs) a partir de tecnologías del CSIC, bien como spin-offs de los centros o bien a través de iniciativas de emprendedores externos al CSIC que se vinculaban a las tecnologías del CSIC mediante los consiguientes contratos de licencia o contratos de desarrollo. Para mejorar la coordinación entre las actividades de promoción y licencia de tecnologías con la creación de EBTs, esta unidad ha desaparecido como tal y desde abril del 2012 el Departamento de Comercialización ha asumido sus tareas.

El procedimiento que utiliza el CSIC para proteger sus tecnologías se ha ido refinando con el tiempo, y ha sido fruto de una larga experiencia de interacción con las empresas de diferentes sectores, la implantación de políticas de gestión adaptadas de otras experiencias internacionales, el paulatino incremento del número de personas con alta cualificación que trabajan en la VATC y de su posterior capacitación complementaria. Dicho procedimiento de gestión de las solicitudes de patente está definido por los siguientes pasos:

- Detección del conocimiento generado en los centros del CSIC y evaluación sobre su posible aplicación industrial y protección mediante patente.

- Elaboración y presentación de la solicitud de patente española (solicitud prioritaria) en la Oficina Española de Patentes y Marcas (OEPM, 2012A).

- Promoción activa de la tecnología protegida por la solicitud de patente como oferta tecnológica entre empresas del sector industrial de aplicación. El interés mostrado por el sector productivo durante esta promoción se tiene en cuenta a la hora de decidir la extensión de los derechos de patente a otros países, mediante solicitud internacional PCT, antes del término del año de prioridad de la patente. La solicitud internacional PCT permite extender los derechos de explotación de la solicitud de patente en los más de 130 países firmantes del tratado PCT durante un periodo de tiempo que finaliza al cumplirse el mes 30 desde la fecha de solicitud de la patente española prioritaria.

- Una vez finalizado el periodo PCT, las patentes del CSIC entran en las fases nacionales y regionales en los casos en los que (i) la solicitud de patente está licenciada, o (ii) la tecnología es estratégica para la institución y se considera que su puesta en el mercado requiere de un periodo de tiempo más amplio que el proporcionando por la PCT.

La toma de decisiones relativas a la entrada en PCT o fases nacionales de las diferentes solicitudes de patente se realiza examinando y evaluando toda la información relativa a cada patente, incluyendo las muestras de interés de empresas, la existencia de documentos que pueden afectar a la concesión de la misma detectados a partir de las búsquedas realizadas en diversas oficinas de patentes, etc. Las decisiones se toman teniendo en cuenta la opinión del inventor, así como su conocimiento del sector de la técnica, y manteniendo siempre un contacto fluido con éste.

Todo este protocolo de actuación que se aplica sobre la selección de solicitudes de patentes para su posterior protección ha tenido un éxito notable. El CSIC es la primera entidad generadora de patentes nacionales (OEPM, 2012B) y, por otro lado, las bases de datos de la OMPI (WIPO, 2012), señalan que el primer solicitante español de solicitudes de patente PCT en el año 2010 es el CSIC en el puesto 122, con 126 solicitudes. La figura 2 a muestra el número de solicitudes de patentes prioritarias registradas en la OEPM y las de tipo PCT presentadas por el CSIC entre 1999 y 2011, junto con 
el número de patentes que en cada año entraron en fases nacionales o regionales en algún país. Se puede observar como el número de solicitudes de patente de prioridad del CSIC ha sufrido un aumento no lineal con el paso de los años, experimentando un fuerte crecimiento tanto en el periodo 1998-2002 como en los últimos cuatro años en los que se ha pasado de una media de 110-120 patentes/año a 170-180 patentes/año. Las solicitudes de patentes en año de prioridad y en el periodo PCT, junto con aquellas que se encuentran en fases nacionales y/o fase europea pero sin haberse licenciado, constituyen la "cartera de patentes" en comercialización del CSIC.

Además, con la intención de concentrar una mayor cantidad de recursos en la promoción de patentes en el sector productivo, la política de la VATC en los últimos años ha sido la de incrementar el personal altamente cualificado, preferiblemente doctores con experiencia en la empresa privada o en otras entidades como oficinas de patentes o en la Comisión Europea, conocedores por lo tanto de las peculiaridades del mundo de la investigación y las del sector productivo. Con esta filosofía se lanzó el Programa JAE-TRANSFER, que permitió en 2010 la contratación de 16 doctores que se incorporaron a otros tantos centros del CSIC bajo la supervisión y coordinación del Departamento de Comercialización de la VATC con el fin de apoyar y gestionar la transferencia de tecnología de dichos centros lo que ha permitido reforzar con más de 25 técnicos de media en los últimos 2 años la ejecución de estas actividades.

Las actividades de promoción de las tecnologías del CSIC, que se han potenciado a través de Departamento de Comercialización de la VATC en estos últimos años, han sido, fundamentalmente:

- La participación en ferias tecnológicas nacionales e internacionales que permiten mostrar las tecnologías del CSIC a las empresas asistentes a las mismas, además de permitir conocer el estado de la técnica y nivel de desarrollo del sector industrial y de facilitar la promoción internacional de la institución.

- La actualización permanente de la página web de la VATC del CSIC (CSIC-Oferta, 2012), en la que se incluyen todas las ofertas tecnológicas procedentes de las patentes presentadas por el CSIC para proteger su actividad investigadora.

- La búsqueda proactiva de empresas en los sectores de posible aplicación de la tecnología usando bases de datos, páginas web de las propias empresas, de asociaciones empresariales, plataformas tecnológicas, etcétera.

- Uso de las herramientas proporcionadas por la red europea EEN, Enterprise Europe Network (EEN, 2012).

El papel de la red EEN debe ser destacado. Dicha red está financiada por la Comisión Europea, dentro del Programa Marco de Competitividad e Innovación (CIP), en el que participan los 27 países miembros de la Unión Europea, además de 15 países que no pertenecen a la UE, entre ellos México, Chile, Turquía, EE.UU, China, Japón, Rusia y Corea del Sur, que pueden participar en el mismo sin recibir financiación. Entre los objetivos de la red está el fomento de la transferencia de tecnología trasnacional, entre dos o más países de la red, uniendo oferta y demanda tecnológica. En la red participan más de 500 instituciones a través de los nodos o consorcios existentes en todas las regiones europeas, y en los países no europeos que participan en la red. El CSIC es socio de la red a través de su nodo en Madrid, y utiliza tres de las herramientas de esta red para promocionar su oferta tecnológica en el extranjero: 
- El sistema Business Boletín System (BBS), que es la base de datos de oferta y demanda tecnológica más importante del mundo, en la que las instituciones que conforman la red vuelcan toda la información sobre oferta y demanda tecnológica de instituciones o empresas de la región donde la institución está ubicada. El CSIC incluye su oferta tecnológica (tecnologías protegidas habitualmente mediante patentes) en esta base de datos, y realiza búsquedas de demandas tecnológicas que encajen con las mismas.

- La herramienta Automatic Matching Tool, que es un sistema de alerta ligada a la BBS, donde se recibe, semanalmente, un boletín con toda la oferta y demanda tecnológica promocionada durante esa semana en la red, así como noticias de interés en las áreas científicotecnológicas seleccionadas.

- Jornadas de transferencia de tecnología. Estas jornadas se suelen organizar ligadas a ferias, congresos u otro tipo de eventos internacionales y suelen durar uno o dos días. En estas jornadas se ponen en contacto ofertantes de tecnología (fundamentalmente Organismos Públicos de Investigación y universidades) y demandantes de tecnología (empresas), con el fin de que ambos puedan llegar a acuerdos de transferencia de tecnología.

El interés del Departamento de Comercialización de la VATC es establecer una relación de confianza con las diferentes empresas contactadas a través de estas iniciativas, de forma que establezca un intercambio de información entre la VATC y la empresa con una regularidad determinada. De este modo las empresas tienen acceso a las últimas tecnologías desarrolladas en el CSIC, y éste tiene la oportunidad de promocionar las mismas, así como de obtener información del sector productivo sobre las posibilidades de sus tecnologías en el mercado, lo que resulta de gran utilidad para tomar decisiones referentes al mantenimiento de solicitudes de patentes y a la propia orientación de los proyectos de investigación del CSIC.

La figura $2 \mathrm{~b}$ muestra el número total de patentes y otro tipo de objetos protegidos que han sido licenciados por el CSIC en los últimos diez años. Esta figura permite constatar el éxito de las estrategias de comercialización desarrolladas en el CSIC. El número de patentes licenciadas por año oscila entre 15 y 70, con una media de 41 patentes licenciadas al año en los últimos 10 años. En particular se puede observar como en el año 2011 el número de patentes licenciadas aumentó en más de un 100\% con respecto del año anterior. Este espectacular incremento es seguramente debido a la puesta en marcha del plan de promoción directa de las ofertas tecnológicas en el CSIC, así como al aumento de personal dedicado a estas actividades a través del Programa JAE-TRANSFER antes mencionado. Por lo tanto, en una primera aproximación se puede inferir que el factor más determinante para mejorar las cifras de patentes licenciadas está relacionado con la presencia de capital humano cualificado que el CSIC dedica a las tareas de promoción del conocimiento generado y protegido por la institución.

Para evaluar la influencia que tiene la promoción de las tecnologías en su posterior licencia, la figura 3 muestra el número de expresiones de interés (EoI) recibidas en los últimos 8 años a través del sistema BBS de la Enterprise Europe Network, y el número de patentes licenciadas por año. Se puede apreciar que las tareas de promoción realizadas en los últimos años se han traducido en un aumento del número de EoI recibidas y, a su vez, del número de patentes licenciadas. Estos datos muestran la relevancia que tiene la promoción activa de tecnologías en la transferencia de tecnología de centros públicos de investigación a empresas privadas. 
FIGURA 2. (a) Número de solicitudes de patentes del CSIC desde el año 1999, indicando las patentes prioritarias (columna azul), las solicitudes internacionales PCT (columna roja) y las patentes que han entrado en fases nacionales y/o regionales (columna verde). (b) Evolución del número de objetos protegidos mediante patentes u otras formas de protección industrial (secretos industriales, materiales biológicos, etc) que fueron licenciadas por el CSIC durante los últimos 10 años.
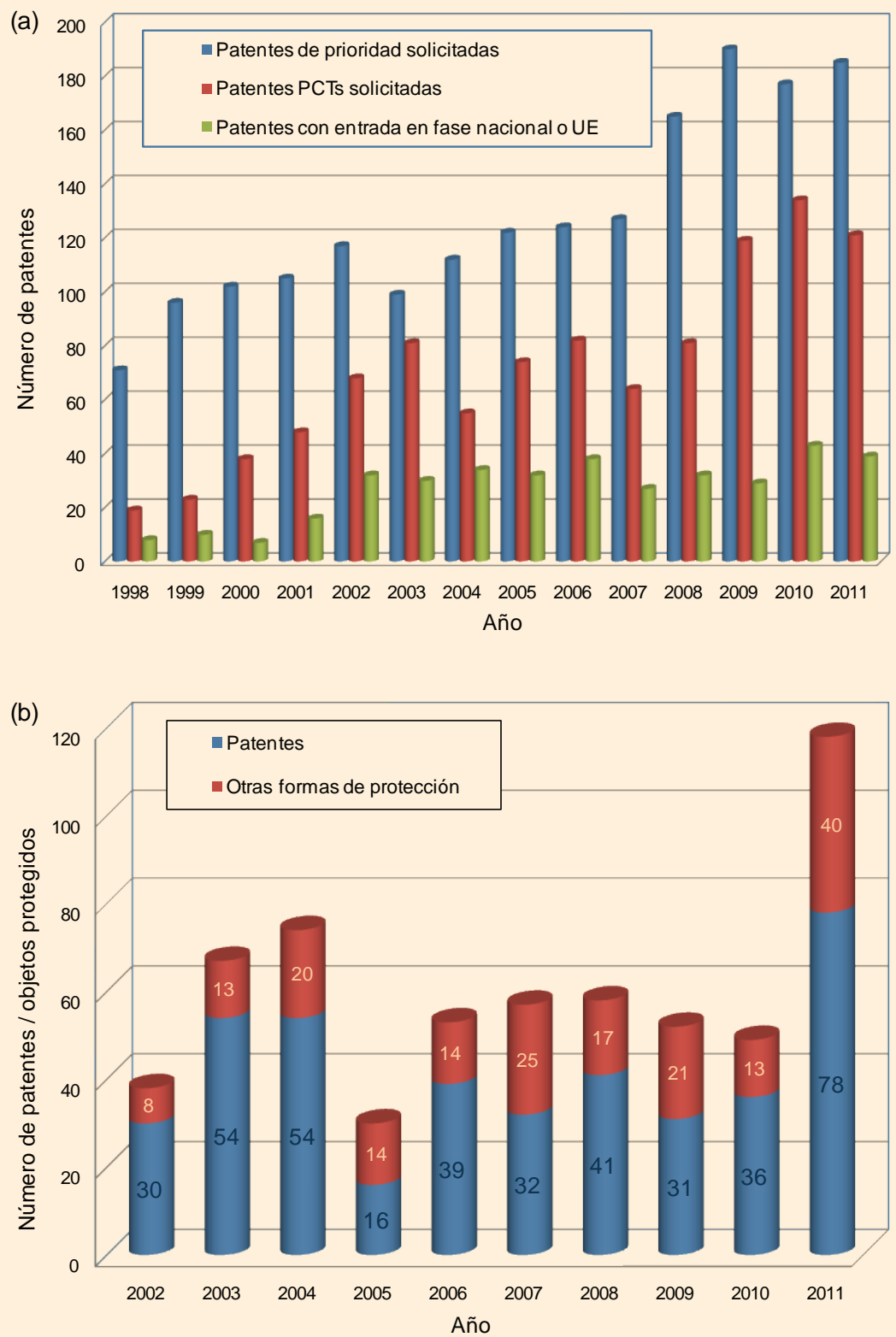
FIGURA 3. Evolución del número de expresiones de interés (Eol) recibidas a través del sistema BBS de la Red Enterprise Europe Network (EEN) y de las patentes licenciadas por el CSIC en el periodo 2004-2011. Las líneas sirven para ilustrar las tendencias en los periodos 2004-2008 y 2009-2011.

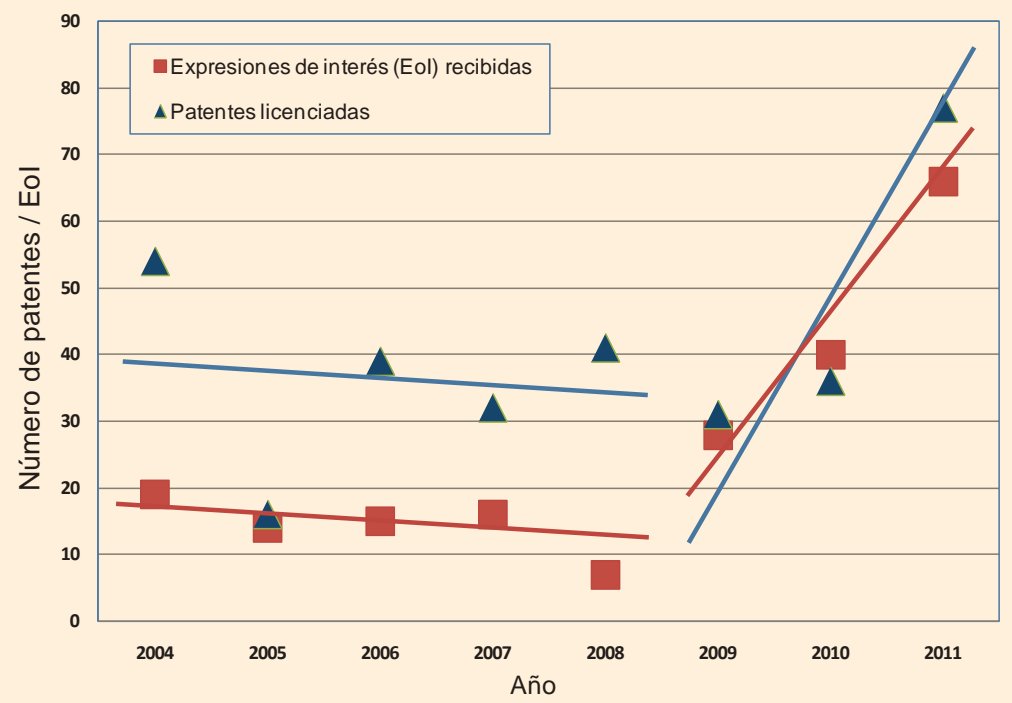

\section{ComercializaCión de la nanOteCnOlogía del CSIC}

El auge que ha tenido la nanotecnología en la última década se ha propagado a la actividad científica en el CSIC y, evidentemente, tiene reflejo en la cartera tecnológica de esta institución. Así, la figura 4 muestra el aumento del número de solicitudes de patentes de prioridad en nanotecnología en el CSIC en los últimos 14 años. Este crecimiento tiene dos componentes, por un lado una componente externa, propiciada por el aumento natural que ha experimentado el tópico emergente de la nanotecnología en el contexto mundial, pero por otro lado hay un factor interno, que es especialmente llamativo a partir del año 2008, fecha en la que la VACT puso en marcha las diversas actuaciones descritas en la sección anterior. Hay que recordar también que la nanotecnología es un campo multidisciplinar y precisamente la estructura interna del CSIC favorece las sinergias entre grupos de diferentes disciplinas.

Es cierto que estas estrategias de valorización y comercialización se han aplicado a todos los sectores de conocimiento, por lo que se podría pensar que el aumento del número de solicitudes de patentes de nanotecnología en el CSIC es consecuencia del aumento del propio número total de solicitudes de patentes en el CSIC en los últimos años. Sin embargo, desde 2006 se comenzaron desde la VATC tareas de prospección y seguimiento de nuevos grupos de investigación del sector de materiales o ciencias físicas, tareas que se dirigieron especialmente también a grupos de investigación del sector "nano" a partir de 2008. En la figura 4 también se muestra que el porcentaje de solicitudes de patentes de nanotecnología con respecto al número total de solicitudes de patentes del CSIC ha ido aumentando con los años, pasando de ser menos del $2 \%$ a finales de los años noventa, a estar por encima del $16 \%$ en los últimos cuatro 
FIGURA 4. Número de solicitudes de patentes de prioridad en el área de la nanotecnología presentadas por el CSIC entre los años 1998 y 2011 . El número sobre cada columna indica el porcentaje de solicitudes de patentes de prioridad de nanotecnología con respecto al número total de solicitudes de patentes de prioridad presentadas por el CSIC ese año (dato mostrado en la columna azul de la Figura $2 a$ ).

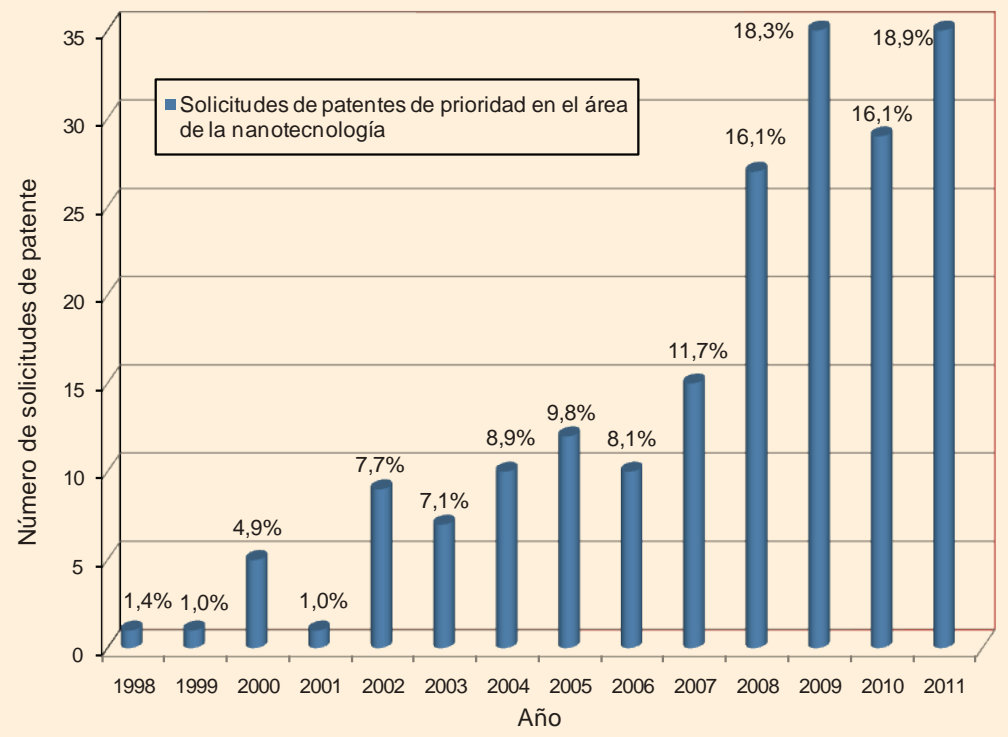

años. Por tanto, cada vez se desarrollan más investigaciones en nanociencia y nanotecnología en el CSIC, que se traducen en un mayor peso de esta actividad en la cartera de tecnología de la institución. Además, hay que destacar que el éxito en el registro de patentes, su comercialización y su posterior licencia, tiene un efecto llamada para otros investigadores del CSIC, que realizan brillantes investigaciones en el tema, pero que inicialmente son reacios a iniciar una andadura que les puede suponer mucho esfuerzo y en la que tienen cierta desconfianza antes las pocas expectativas de éxito. Esta dinámica puede permitir que el número absoluto de las solicitudes de patentes y el de patentes licenciadas de nanotecnología del CSIC (y su peso relativo) sigan creciendo en los próximos años.

La figura 5 muestra las diferentes áreas de aplicación de las solicitudes de patentes de nanotecnología del CSIC. Se puede observar un aumento considerable del peso de las solicitudes de patente con aplicación en el área de los nuevos materiales y de la energía. Sin embargo, el número de patentes con aplicación en el área de biotecnología se ha mantenido constante y, por tanto, su peso en la cartera tecnológica de nanotecnologías del CSIC ha disminuido considerablemente a medida que ésta aumentaba. Otras aplicaciones como las del sector químico han mantenido su peso en la cartera tecnológica. Este gráfico ilustra el amplio abanico de aplicaciones de la nanotecnología que se constituye, de hecho, como un gran conjunto de herramientas y técnicas, de muy diversa procedencia, pero que tiene un carácter de elemento facilitador transversal en el que se van a apoyar otros desarrollos tecnológicos. De hecho, en el próximo Programa Marco de Investigación e Innovación de la Unión Europea, denominado "Horizon 2020" (Horizon2020, 2012) la nanotecnología aparece como una de las seis 
FIGURA 5. Clasificación, según el área de aplicación, de las solicitudes de patentes de prioridad del CSIC en nanotecnología presentadas entre los años 1998 y 2011.

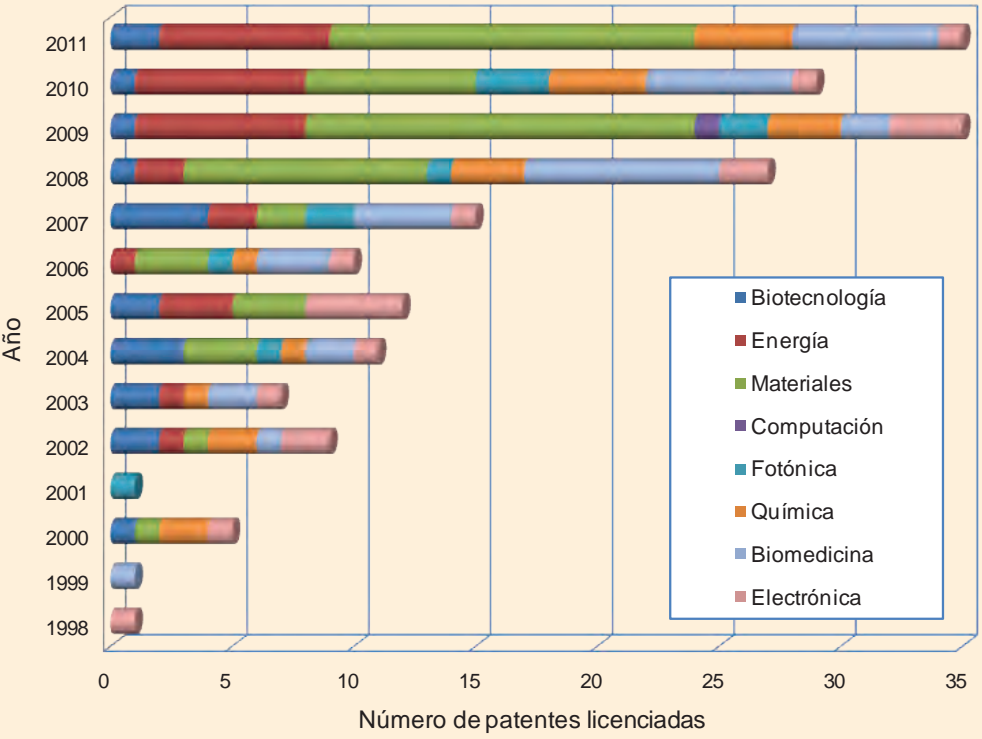

Tecnologías Claves KET (key enabling technologies) que pueden asegurar el nivel de liderazgo de la industria europea.

En línea con el aumento del peso de la nanotecnología en la contribución tecnológica del CSIC, la promoción de la cartera de nanotecnología del CSIC se ha considerado estratégica dentro de la VATC del CSIC. De hecho, las principales herramientas de promoción desarrolladas en la VATC en los últimos años se han aplicado, con mayor estímulo y constancia, al sector "nano", para posteriormente trasladarse a otras áreas tecnológicas como alimentación o energía. En el año 2008, el CSIC, a través de la VATC, decidió participar en las ferias de nanotecnología más importantes del mundo, y dirigir parte de su actividad de promoción hacia el mercado asiático, habida cuenta del enorme desarrollo que la nanotecnología ha tenido en los países del este asiático, y su gigantesco potencial de mercado. Desde entonces, el CSIC ha participado en un total de 10 ferias tecnológicas del sector de la nanotecnología en 7 países diferentes de Europa y Asia, todas ellas de reconocido prestigio internacional. En la tabla I, se muestran todos los datos sobre dichas participaciones, incluyendo el número de ofertas tecnológicas incluidas en el catálogo y el número de empresas con las que se establecieron entrevistas. La cartera tecnológica que se ha promocionado ha estado constituida por una media de 24 ofertas tecnológicas/feria en constante renovación (fluctuando entre 16 y 32 patentes). La cartera tecnológica del CSIC en nanotecnologías se ha nutrido de las patentes generadas en 21 centros de investigación del CSIC situados en Barcelona, Madrid, Oviedo, Sevilla, Valencia y Zaragoza.

Entre las ferias de nanotecnología en las que ha participado la VATC del CSIC cabe destacar la participación, durante cuatro años consecutivos (2009-2012), en 
TABLA I. Participación del CSIC en ferias internacionales de nanotecnología (2009-2012)

\begin{tabular}{|c|c|c|c|c|}
\hline $\begin{array}{c}\text { Nombre de la feria } \\
\text { o evento }\end{array}$ & Fecha & Lugar & $\begin{array}{l}\text { Número de } \\
\text { ofertas en el } \\
\text { catálogo CSIC }\end{array}$ & $\begin{array}{l}\text { Número de } \\
\text { reuniones con } \\
\text { empresas }\end{array}$ \\
\hline Nano Tech 2009 & 18-20 febrero 2009 & Tokio (Japón) & 28 & 29 \\
\hline $\begin{array}{l}\text { Euronanoforum } \\
2009\end{array}$ & 2-5 junio 2009 & Praga (República Checa) & 16 & 15 \\
\hline $\begin{array}{l}\text { Taiwan Nano } \\
\text { Exhibition } 2009\end{array}$ & 7-9 octubre 2009 & Taipei (Taiwán) & 20 & 10 \\
\hline Nano Tech 2010 & 17-19 febrero 2010 & Tokio (Japón) & 24 & 17 \\
\hline $\begin{array}{l}\text { Micronano System } \\
\text { Workshop } 2010\end{array}$ & 4-5 mayo 2010 & Estocolmo (Suecia) & 18 & 11 \\
\hline $\begin{array}{l}\text { Taiwan Nano } \\
\text { Exhibition } 2010\end{array}$ & 7-9 octubre 2010 & Taipei (Taiwán) & 29 & 7 \\
\hline Nano Tech 2011 & 16-18 febrero 2011 & Tokio (Japón) & 32 & 38 \\
\hline ImagineNano 2011 & 11-14 abril 2011 & Bilbao (España) & 29 & 31 \\
\hline Rusnanotech 2011 & 26-28 octubre 2011 & Moscú (Rusia) & 19 & 13 \\
\hline Nano Tech 2012 & 15-17 febrero 2012 & Tokio (Japón) & 27 & 25 \\
\hline
\end{tabular}

la feria más importante del mundo "Nano Tech Tokyo" (Nanotech, 2012) que se celebra todos los años en el mes de febrero en Tokio (Japón). En estas ferias la VATC participó mediante un módulo expositivo dentro del Pabellón Español, cofinanciado por el Instituto de Comercio Exterior (ICEX) (ICEX, 2012) y coordinado por la Fundación Phantoms (Phantoms, 2012). Tal y como se puede observar en la tabla I, la VATC del CSIC también participó en la feria de nanotecnología de Taiwán, país en el que la nanotecnología ha experimentado un enorme crecimiento y que cuenta con una excelente planificación en cuanto a la formación de recursos humanos, transferencia de tecnología, creación de empresas, etc. La participación del CSIC en la feria Taiwan Nano Exhibition (TaiwanNano, 2012) se realizó tanto con un stand propio del CSIC en el año 2009 como dentro del Pabellón Español en el año 2010. También cabe destacar la participación del CSIC en la feria RusNanoTech celebrada en octubre del año 2011, aprovechando la celebración del Año Dual España-Rusia (Rusnanotech, 2011). Es importante mencionar que la participación en estas ferias implica un gran esfuerzo previo de contacto con cientos de potenciales clientes y la elaboración de materiales específicos de promoción para cada una de ellas. En la figura 6 se muestran algunos de estos materiales y una imagen de uno de los módulos expositivos del CSIC.

La participación del CSIC en estas ferias dio lugar a un total de 196 reuniones con empresas, y el inicio de negociaciones, tanto para el desarrollo conjunto de proyectos de I+D para acercar las tecnologías al mercado como de licencia de las patentes ofertadas. Otro aspecto que debe resaltarse es que la participación en estas ferias originó contactos entre la VATC y grupos de investigación de diferentes institutos del CSIC que trabajan en nanotecnología. De esta forma, todo este trabajo de promoción a nivel 
FIGURA 6. (a) Cartel publicitario y (b) ejemplo de una ficha tecnológica elaborados para la participación del CSIC en la Feria NanoTech celebrada en Tokio (Japón). (c) Imagen de una reunión celebrada en dicha feria internacional entre representantes de la VATC del CSIC y un representante de una empresa japonesa.

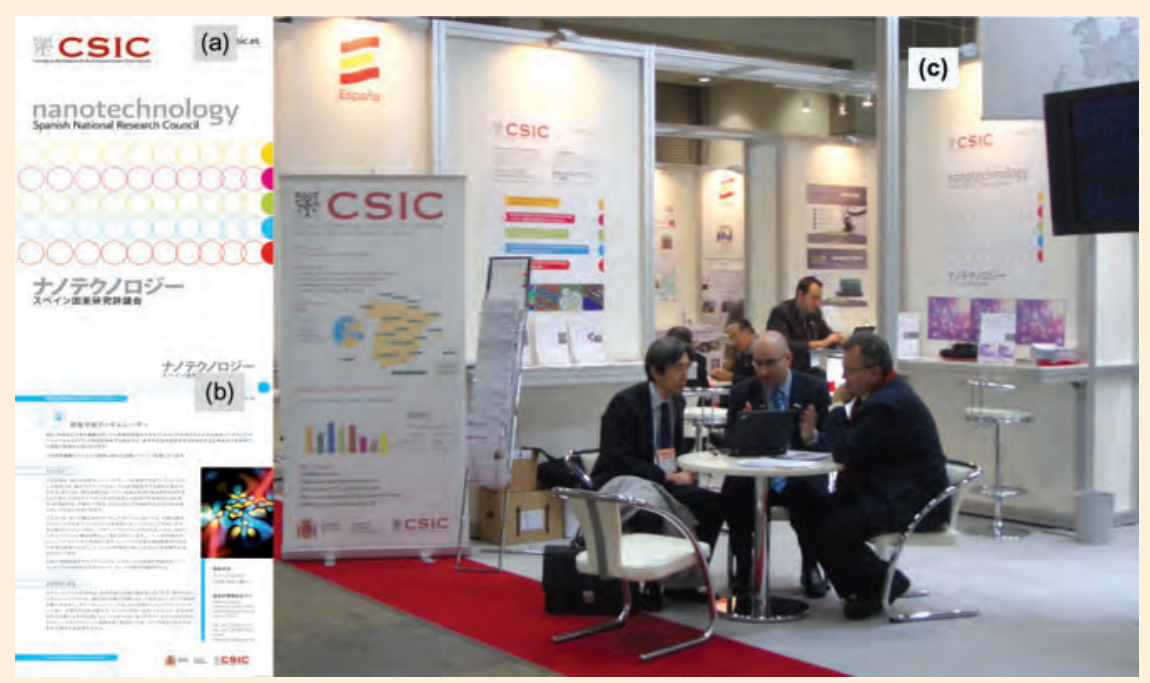

internacional realizado por la VATC del CSIC contribuyó a despertar el interés de los propios investigadores del CSIC en desarrollar y proteger sus invenciones en nanotecnología y, por tanto, ha posibilitado el alto número de solicitudes de patentes del CSIC y la consolidación de una cartera en nanotecnologías en los últimos 4 años con un alto grado de extensión internacional.

La figura 7 muestra el número de patentes licenciadas en el CSIC desde el año 2002 (obsérvese que no existen licencias de patentes del CSIC en nanotecnologías antes de dicho año). Se puede observar un aumento progresivo del número de licencias de patentes de nanotecnología del CSIC hasta el año 2010, en el que hay un descenso considerable y, posteriormente, en el 2011 un aumento muy significativo. El descenso ocurrido en el año 2010 es consecuencia, posiblemente, de la dedicación por parte del personal de la VATC a la formación de los técnicos contratados ese año mediante el programa JAE-TRANSFER hasta que se incorporaron a los diferentes institutos o centros. Sin embargo, al año siguiente, con toda esta plantilla ya formada y los esfuerzos en comercialización que se estaban llevando a cabo, se alcanzó el número de 19 patentes licenciadas en el área de la nanotecnología. Este número de licencias alcanzado hubiera sido impensable años atrás. Estas licencias de patentes se firmaron con empresas de Alemania, España, Suecia, Francia, EEUU y Reino Unido, y han aportado al CSIC retornos directos en forma de down-payment y aseguran un porcentaje de los futuros ingresos por ventas netas de los productos protegidos mediante esas patentes. 
FIGURA 7. Solicitudes de patentes de prioridad en nanotecnología que han sido licenciadas por el CSIC durante el periodo 2002-2011. Las patentes han sido clasificadas por su sector de desarrollo en dos grupos, por un lado biotecnología y salud (etiqueta "BIO") y por otro las que corresponden a aplicaciones de la nanotecnología en sectores no relacionados con biotecnología o salud (etiqueta "NO BIO").

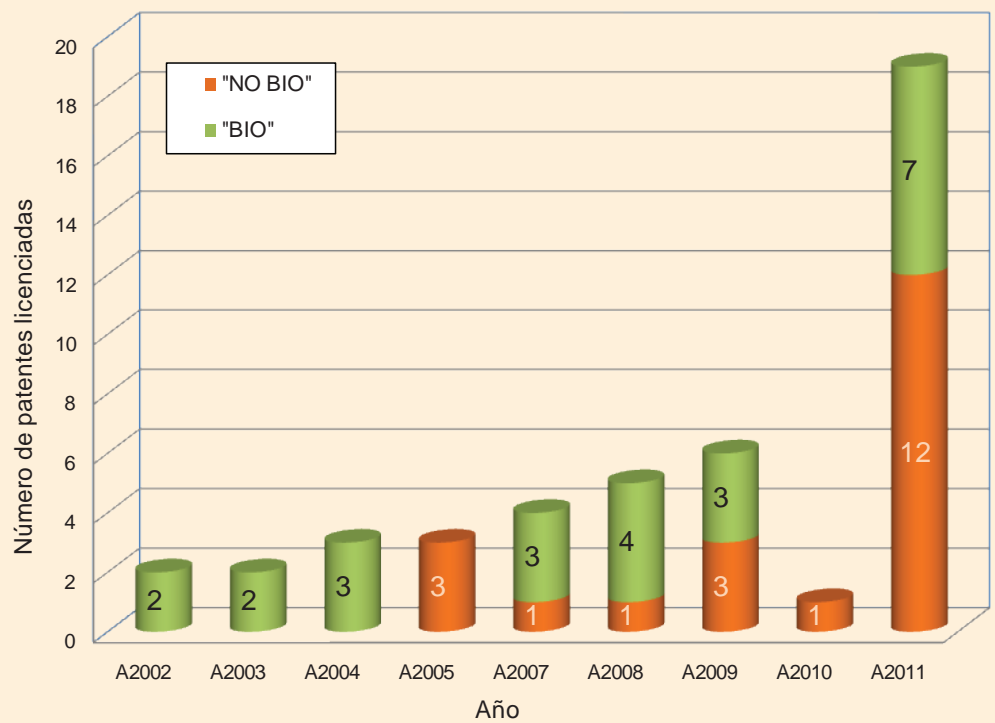

\section{Conclusiones}

En estos momentos no hay ninguna duda sobre el fuerte impacto que la nanotecnología va a tener en diversos sectores económicos en los próximos años. La primera década del siglo XXI ha sido testigo del salto desde la nanociencia hasta la nanotecnología, que se ha comenzado a implementar en productos que encontramos cada vez con más frecuencia en el mercado. Esta transición ha sido posible gracias a la puesta en marcha de distintas estrategias que han requerido una ingente cantidad de recursos. EEUU, China, Japón, y Alemania son los países que están liderando esta "nano-revolución", aunque prácticamente todos los países que realizan actividades de I+D han seguido su estela, asumiendo que la nanotecnología es un elemento estratégico del desarrollo tecnológico que tendrá lugar en el siglo XXI.

Se ha pasado de un contexto en el que las publicaciones eran el producto más evidente de la nanociencia a otro, marcado por la imperiosa necesidad de convertir todo ese conocimiento, que sigue creciendo a buen ritmo, en bienes, productos y servicios. En los últimos años se ha asistido a un espectacular crecimiento del número de patentes en nanotecnología, la aparición de cientos de empresas de base nanotecnológica y la irrupción de miles de "nanoproductos" en los mercados. Estos indicadores, vinculados a la transferencia de la nanotecnología hacia los sectores productivos, se sustentan en la actividad desarrollada en los países más avanzados y aquellos con economías emergentes. Sin embargo, los países con menos tradición o capacidad industrial corren el riesgo de no posicionarse bien en el mercado internacional de las aplicaciones de la nanotecnología. 
España está en una situación intermedia en el concierto internacional, de forma que, tras haber encontrado un hueco entre las naciones que consideran que la investigación es un tema de capital importancia y haber conseguido una cuota razonable de participación en la producción científica internacional, no ha encontrado aún la manera efectiva de convertir el conocimiento que genera a través de actividades de I+D en el soporte necesario para promover una poderosa industria competitiva a nivel internacional. Se puede afirmar que, en general, se ha desarrollado una legislación adecuada, y se han llevado a cabo unas políticas razonables de financiación de la I+D, promoviendo la actividad investigadora tanto en centros de investigación como en la industria, y constituyendo diferentes estructuras de interfaz entre el entorno académico y el productivo, como son las oficinas especializadas en transferencia de tecnología, los parques científicos y tecnológicos, etc. Sin embargo, no parece que todas estas actuaciones hayan sido suficientes para impulsar la innovación en la industria española sobre la base del conocimiento generado por el sistema nacional de investigación y desarrollo. Este contexto del sistema ciencia-tecnología-sociedad es en el que la nanotecnología ha aparecido en España, con su virtudes y sus defectos. Entre las virtudes podemos destacar una buena producción científica con un impacto razonable y una buena cosideración de muchos investigadores españoles en el contexto mundial Entre los defectos podemos mencionar una cierta descoordinación entre los agentes y la ya mencionada ausencia de una transferencia efectiva de conocimiento hacia el sector productivo.

En general, la situación del CSIC, mayor organismo público de investigación de este país, es similar a la que presenta el conjunto del sistema público de investigación. Sin embargo, en lo referente a la transferencia del conocimiento, el CSIC ha puesto en marcha, desde hace 5 años, una estrategia que pretende no sólo incentivar las solicitudes de patentes, sino lograr que estas tengan un éxito real, transfiriéndolas al sector productivo, tanto nacional (preferentemente) como extranjero. Esta estrategia se ha ido mejorando en función de la experiencia y tiene como principal objetivo reforzar las actividades destinadas a la comercialización de las patentes mediante el uso de diferentes herramientas de gestión, la participación en redes internacionales, y la interacción con empresas en las ferias tecnológicas más importantes. Los programas de contratación y formación de expertos en transferencia han sido clave para incentivar en los centros la transformación del conocimiento en patentes, y para proyectar estas patentes en el sector productivo nacional e internacional. En el caso de la nanotecnología, la estrategia del CSIC ha resultado especialmente exitosa, tanto en número de patentes presentadas como en número de patentes licenciadas.

El entorno económico y presupuestario se ha ensombrecido en estos últimos años, y es muy probable que algunas de las iniciativas y programas que se habían puesto en marcha para fomentar la transferencia de tecnología no puedan sostenerse por falta de recursos. En este contexto será complicado que los datos exitosos que hemos mostrado en las figuras anteriores se mantengan. Sin embargo se deben buscar soluciones para evitar que esto suceda y seguir potenciando una de las funciones fundamentales del CSIC: transferir el conocimiento generado y las tecnologías desarrolladas mediante financiación pública al mercado para solucionar problemas concretos y mejorar el nivel del vida de los ciudadanos, así como para potenciar el tejido industrial y crear nuevas empresas y puestos de trabajo de calidad, asegurando así un retorno económico del dinero público invertido en investigación y desarrollo. El ejemplo que se ha expuesto aplicado a la nanotecnología en el CSIC muestra que se 
pueden lograr muy buenos resultados en transferencia de tecnología, siempre que se tengan los medios apropiados y éstos se organicen adecuadamente.

Los autores desean que este trabajo, que relata de manera somera la situación de la nanotecnología y su transferencia en España en general, y en el CSIC en particular, sirva de fuente de inspiración para promover modelos similares, adaptados o mejorados, en otras oficinas o departamentos de centros de investigación dedicados a la promoción de la transferencia de la nanotecnología.

\section{Agradecimientos}

Quisieramos agradecer a los responsables y a todo el personal de la VATC el esfuerzo y dedicación realizado durante todos estos años. También debemos destacar que la colaboración de muchos investigadores del CSIC en las actividades de transferencia y comercialización ha contribuido a alcanzar con éxito los resultados aquí presentados. Asimismo, agradecemos a la Comisión Europea la financiación que ha recibido el CSIC dentro de la red Enterprise Europe Network, contribuyendo así a promocionar la cartera tecnológica del CSIC en Europa, y facilitando la transferencia de tecnología transacional. P.A. Serena quiere agradecer al Ministerio de Economía y Competitividad el apoyo a través del proyecto ACI2010-1134 "Promoción de la cooperación en Nanotecnología con Rusia, Australia y Asia (NANORAA)" y a la Red NANODYF del Programa CYTED.

\section{Bibliografía}

ALBA. (2012). Laboratorio de Luz de Sincrotrón (ALBA). En: <http://www.cells.es/>.

Altran. (2012). Índice Altran de Potencial Innovador 2102. Subínidce 7. Patentes e Innovación en la Unión Europea. En: <http://www.altran.es/fileadmin/medias/ES.altran. es/documents/Indice_Innovacion/Subindices/Subindice_Innovacion7.pdf> .

CEI. (2012). Campus de Excelencia Internacional UAM+CSIC. En: <http://campusexcelenciauam.es/>.

Chacón, C., Estevao, V., Narros, C., Correia, A., Serena, P.A. (2011). Nanotechnology in Spain: Current situation and future challenges, Convertech \& E-Print, 1(6): 26-32.

Cientifica. 2011. Global funding of nanotechnologies \& its impact. July 2011. Cientifica Ltd. En: <http://cientifica.com/wp-content/uploads/downloads/2011/07/Global-Nanotechnology-Funding-Report-2011.pdf>.

Comisión Europea. (2004). Hacia una estrategia europea para las nanotecnologías, Comisión Europea COM (2004) 338. En: <http://ec.europa.eu/nanotechnology/pdf/ nano_com_es.pdf>.

Comisión Europea. (2005). Some Figures about Nanotechnology R\&D in Europe and Beyond, Comisión Europea, Bruselas. En: <ftp://ftp.cordis.europa.eu/pub/nanotechnology/ docs/nano_funding_data_08122005.pdf>.

Correia, A., Sáenz, J.J., Serena, P.A. (2006). El lento despertar de la nanotecnología en España, Revista Sistema Madri+d 15 (abril 2006): 3-7. En: <http://www.madrimasd.org/revista/revista35/editorial/editorial.asp>. 
Correia, A., Serena, P.A. (2009). 2010-2020: ¿la década del despegue de la nanotecnología española? Física y Sociedad 20: 36-39. En: <http://www.cofis.es/pdf/fys/fys20/ fys20_36-39.pdf>.

Correia, A., Serena, P.A. (2012). Nanotechnology in Spain: from basic science to the market, Asociación Arabinnova. En: <http://hercules.learningclass.com/author/portal/ links/73792_LINK_Pedro\%20Serena-borrador.pdf>.

CSIC. (2006A). Plan de Actuación Institucional del CSIC 2006-2009, Consejo Superior de Investigaciones Científicas. En: <http://www.csic.es/web/guest/plan-de-actuacion-2006-2009>.

CSIC. (2006B). Eje de Nanociencia y Nanotecnología, Consejo Superior de Investigaciones Científicas. En: <http://www.nanored.org.mx/documentos/EjeNano.pdf>.

CSIC. (2010). El CSIC en cifras, Consejo Superior de Investigaciones Científica. En: <http:// newsletters.baetica.es/CSIC/CSIC_DATOS_2010/CSIC_Datos2010.html>.

CSIC. (2012). Consejo Superior de Investigaciones Científicas. En: <http://www.csic.es>.

CSIC-Oferta. (2012). Página web con la oferta tecnológica del CSIC actualizada y presentada por sectores de aplicación. En: <http://www.csic.es/web/guest/oferta-tecnologica>.

Dang, Y., Zhang, Y., Fan, L., Chen, H., Roco, M.C. (2010). Trends in worldwide nanotechnology patent applications: 1991 to 2008, J. Nanopart Res. 12: 687-706.

Delgado, G. C. (2008). Guerra por lo invisible: negocio, implicaciones y riesgos de la nanotecnología, Ceeich, UNAM, México.

Delgado, G. C. (2009). Economía Política de la Nanotecnología, Mundo Nano 1(1): 87-94.

E2I. (2011). Estrategia Estatal de Innovación, Ministerio de Economía y Competitividad (MINECO). En: <http://www.cdti.es/recursos/publicaciones/archivos/33083_57572010144235.pdf>.

Echeverría, J. (2005). Gobernanza de las nanotecnologías, Arbor, 181, núm. 715: 301-315. (doi:10.3989/arbor.2005.i715.414).

EEN. (2012). Enterprise European Network. En: <http://www.enterprise-europe-network.ec.europa.eu/index_en.htm>.

EPO. (2011). Nanotechnology and Patents, European Patent Office. En: <http://www.epo. org/service-support/publications/issues/nanotechnology.html>.

EPO. (2012). European Patent Office. En: <http://www.epo.org/>.

Horizon2020. (2012). En: <http://ec.europa.eu/research/horizon2020/index_en.cfm>.

Hullmann, A. (2006). Who is winning the global nanorace?, Nature Nanotech. 1: 81-83.

Hwang, D. (2010). Ranking the nations on nanotech, Lux Reseach. En: <http://www.electroiq.com/articles/stm/2010/08/ranking-the-nations.html>.

ICEX. (2012). Instituto Español de Comercio Exterior. En: <http://www.icex.es>.

ICONO. (2012). Indicadores del Observatorio Español de I+D+I para la Competitividad (ICONO), Fundación Española de Ciencia y Tecnología (FECYT). En: <http://icono.fecyt. es/indicadores/Paginas/default.aspx?ind=1>.

INE. (2010). Encuesta sobre la Innovación en las empresas, realizada por el Instituto Nacional de Estadística. La información está disponible en: <http://www.ine.es/jaxi/ menu.do?L=0\&type=pcaxis\&path=\%2Ft14/p061\&file=inebase $>$.

Ingenio. (2010). Programa Ingenio 2010. En: <http://www.ingenio2010.es>.

INIC. (2012). Statistical Report of Production of Nanoscience in Iran, Iran Nanotechnology Initiative Council. En: <http://en.nano.ir/index.php/main/page/17>. 
INL. (2012). International Iberian Nanotechnology Laboratory (INL). En: <http://www. inl.int>.

ISE. (2009). ISE Nanotechnology Index, International Securities Exchange. En: <http:// www.ise.com/assets/documents/OptionsExchange/index_reports/TNY_IMG.PDF>.

Kleike, J.W. (ed.). (2009). National Nanotechnology Initiative: Assessment and Recommendations, Nova Science Pub. Inc., Nueva York.

Ley de la Ciencia. (1986). Ley 13/1986, de 14 de abril, de Fomento y Coordinación General de la Investigación Científica y Técnica, Boletín Oficial del Estado Español 93, pp. 1376713771. En: <http://www.boe.es/boe/dias/1986/04/18/pdfs/A13767-13771.pdf>.

Ley de la Ciencia. (2011). Ley 14/2011, de 1 de junio, de la Ciencia, la Tecnología y la Innovación, Boletín Oficial del Estado Español 131: 54387-54455. En: <http://www.boe. es/boe/dias/2011/06/02/pdfs/BOE-A-2011-9617.pdf>.

LFSPN. (2012). Laboratorio de Física de Sistemas Pequeños y Nanotecnología. En: <http:// www.fsp.csic.es/>.

Lindsay, S. (2009). Introduction to Nanoscience, Oxford University Press.

López, M.S., Hasmy, A., Vessuri, H. (2011). Nanoscience and nanotechnology in Venezuela, J. Nanopart. Res. 13: 3101-3106.

Meri, T. (2009). China passes the EU in High-Tech exports, Eurostat Statistics in Focus 25: 1-8. En: <http://epp.eurostat.ec.europa.eu/cache/ITY_OFFPUB/KS-SF-09-025/EN/ KS-SF-09-025-EN.PDF>.

Merrill Lynch. (2004). Merrill Lynch Nanotech Index, Merrill Lynch. En: <http://www. ml.com/media/42322.pdf>.

MINECO. (2012). Ministerio de Economía y Competitividad. En: <http://www.mineco.gob. es/stfls/mineco/investigacion.htmls.

MINETUR. (2010). Estructura de la Industria por Ramas de Actividad 2010, Ministerio de Industria, Energía y Turismo (MINETUR). En: <http://www.minetur.gob.es/es-ES/ IndicadoresyEstadisticas/DatosEstadisticos/III.\%20Industria/III_10.pdf>.

Mowerya, D. C., Ziedonisb, A. A. (2002). Academic patent quality and quantity before and after the Bayh-Dole act in the United States, Research Policy 31: 399-418.

NanoSpain. (2012). Red Española de Nanotecnología. En: <http://www.nanospain.org>.

Nanotech. (2012). Nano Tech International Nanotechnology Exhibition \& Conference, En: <http://www.nanotechexpo.jp/en/>.

NNI. (2012). National Nanotechnology Initiative. En: <http://www.nano.gov/>.

Noyons, E.C.M., Buter, R.K., van Raan, A.F.J., Schmoch, U., Heinze, T., Hinze, S., Rangnow, R. (2003). Mapping Excellence in Science and Technology across Europe Nanoscience and Nanotechnology, informe del proyecto EC-PPN CT-2002-0001 a la Comisión Europea. En: <ftp://ftp.cordis.europa.eu/pub/indicators/docs/mapex_nano. pdf>.

OEI. (2009). La nanotecnología en Iberoamérica. Situación actual y tendencias, Organización de Estados Iberoamericanos. En: <http://www.oei.es/salactsi/nano.pdf>.

OEPM. (2012A). Oficina Española de Patentes y Marcas. En: <http://www.oepm.es/es/ index.html>.

OEPM. (2012B). Memoria de Actividades 2011. Propiedad Industrial y Empresa, Oficina Española de Patentes y Marcas. En: <http://www.oepm.es/es/sobre_oepm/actividades_estadisticas/memorias_actividades/index.html>. 
OPTI. (2008). Aplicaciones Industriales de las Nanotecnologías en España en el Horizonte 2020, Fundación OPTI y Fundación INASMET-TECNALIA, En: <http://www.navarrainnova.com/pdf/2009/nanoindustrial20200PTI.pdf>.

Pagliaro, M. (2010). Nano-Age: How Nanotechnology Changes our Future, Wiley-VCH.

Palmberg, C., Dernis, H., Miguet, C. (2009). Nanotechnology: an overview based on indicators and statistics. STI working paper 2009/7. Statistical Analysis of Science, Technology and Industry, editado por la Organización para la Cooperación y el Desarrollo Económicos (OCDE). En: <http://www.oecd.org/dataoecd/59/9/43179651.pdf>.

Phantoms. (2011A). Nanoscience and nanotechnology in Spain, editado por la Fundación Phantoms. En <http://issuu.com/phantoms_foundation/docs/libro_nanociencia_1-12_con_portadas $>$.

Phantoms. (2011B). Catalogue of Nanoscience \& Nanotechnology Companies in Spain, Phantoms Foundation. En: <http://www.phantomsnet.net/Resources/Catalogue_Companies.pdf>.

Phantoms. (2012). Fundación Phantoms. En: <http://www.phantomsnet.net>.

PNIDI. (2004). Plan Nacional de I+D+i 2004-2007. En: <http://www.idi.mineco.gob.es/ stfls/MICINN/Investigacion/FICHEROS/Plan_Nacional_Vol_IDoc.pdf $>$.

PNIDI. (2008). Plan Nacional de I+D+i 2008-2011. En: <http://www.idi.mineco.gob.es/ stfls/MICINN/Investigacion/FICHEROS/PLAN_NACIONAL_CONSEJO_DE_MINISTROS.pdfs.

Red OTRI. (2010). Informe de la Encuesta de Investigación y Transferencia de Conocimiento 2010 de las Universidades Españolas, Red de Oficinas de Transferencia de Resultados de Investigación (RedOTRI) dependiente de la Conferencia de Rectores de Universidades Españolas (CRUE). En: <http://www.redotriuniversidades.net> (sección Biblioteca).

Represa-Sánchez, D., Castro-Martínez, E., Fernández de Lucio, I. (2005). Encouraging Protection of Public Research Results in Spain, Journal of Intellectual Property Rights 10: 382-388. En: <http://digital.csic.es/bitstream/10261/9772/1/AR16_2_IPR-267. pdf>.

Roco, M.C., Bainbridge, W.S. (eds.). (2001). Societal Implications of Nanoscience and Nanotechnology, Kluwer Press, Boston (EE.UU.) y Dordrecht (Holanda).

Rodríguez, J., Casani, F. (2007). La transferencia de tecnología en España. Diagnóstico y perspectivas, Economía Industrial 366, pp. 15-22. En: <http://www.minetur.gob.es/ Publicaciones/Publicacionesperiodicas/EconomiaIndustrial/RevistaEconomiaIndustrial/366/15.pdf>.

RusNanoTech. (2011). Exhibición internacional Rusnanotech Expo 2011. En: <http:// www.rusnanoforum.ru/eng/>.

Serena, P.A. (2009A). The implementation of the Action Plan for Nanosciences and Nanotechnologies in Spain (2005-2007), E-Nano Newsletter (Editada por la Fundación Phantoms) 15, 14 (2009). En: <http://www.phantomsnet.net/Foundation/Enano_ newsletter15.php>.

Serena, P.A. (2009B). La implantación de la nanotecnología en España: muchas luces y alguna sombra, Mundo-Nano 2 (2): 74-90. En: <http://www.mundonano.unam.mx/ pdfs/mundonano3.pdf>.

TaiwanNano. (2012). Taiwan Nano Exhibition. En: <http://nano.tca.org.tw/index. php?lang=e $>$. 
Thomson Reuters. 2012. Science Citations Index es un producto de Thomson Reuters. En: <http://thomsonreuters.com/products_services/science/science_products/a-z/ science_citation_index/>.

WIPO. (2011A). International Patent Filings Recover in 2010, World Intellectual Property Organization. En: <http://www.wipo.int/pressroom/en/articles/2011/article_0004.html>.

WIPO. (2011B). PCT. The international Patent System Yearly Review. Developments and performance in 2010, World Intellectual Property Organization. En: <http://www.wipo. int/pct/en/activity/pct_2010.pdf>.

WIPO. 2012. Principales solicitantes PCT, World Intellectual Property Organization. En: $<w w w . w i p o . i n t / e x p o r t / s i t e s / w w w / i p s t a t s / e n / s t a t i s t i c s / p c t / x l s / y_{-}$top_applicants. $x \mid s>$. 